\title{
Effects of the Based Membrane on the Hydrophobicity of Super-hydrophobic PES Membrane and its Structural Properties
}

\author{
Xudong Sun \\ Key laboratory of Hollow Fibre Membrane Materials and Membrane Progress \\ Ministry of Education Tianjin Polytechnic University, Tianjin 300160, China \\ E-mail: sunxudun@126.com
}

\begin{abstract}
Polyethersulphone (PES) was a hydrophobilic material. In order to use the Polyethersulphone (PES) membrane on highly hydrophobic membrane separation process, the PES membrane should be improved its hydrophobicity. In this paper, the super-hydrophobic PES membrane was successfully fabricated via sol-gel method. The chemical composition and the physical micro-structure of fabricated PES membrane surface were analyzed by Fourier-Transform Infrared Spectrometers (FTIR), Scanning Electron Microscope (SEM) and Atomic Force Microscope (AFM). The results showed that the PES membrane was super-hydrophobic, and its contact angle was $154^{\circ}$. The super-hydrophobic PES membrane has the low surface energy matters and the micro/nano-meter protuberance on the membrane surface. Those micro/nano-meter protuberances on membrane's surface contributed for an rms roughness, which was about $350 \mathrm{~nm}$. From the SEM images of the membrane cross-section, the spongy pores and the finger-like pores were found in cortex and intermediate layer, respectively.
\end{abstract}

Keywords: PES, Super-hydrophobic membrane, Contact angle, Surface micro-structure

\section{Introduction}

Wettability is one of the primary surface properties of solid materials, which is mainly governed by both the chemical composition and the geometrical microstructure. There has been increasing interest in the fabrication of super-hydrophobic membrane in recent years because of their widespread potential applications in various industrial fields. The phenomenon of super-hydrophobicity was investigated by Wilhelm Barthlott in detail 10 years ago (Barthlott W., 1997, pp.1-8). He found that the water contact angle of lotus-leaf was more than $150^{\circ}$. Lotus-leaf surface was covered by many papillae's with a diameter in the range of 5-15mm accompanying with smaller protrusions of nano-meter size, micro- and nanometer scale roughness can trap a large amount of air, which largely minimized the real contact area between the leaf and the water droplet. As rain drops fall on the leaf, it will remove dirt at the outer layer of the water droplets (Gu, 2003, pp. 922-925, Feng, 2002, pp.1857-1860 and Zhang, 2009, pp.1371-1376).

As far as we know, polyethersulphone membrane was a high-performance membrane, and has an excellent chemical stability and mechanical strength. PES membrane, which can be easily fabricated by phase inversion method, used to microfiltration, ultra-filtration, dialysis and gas separation membrane process. However, owning to the ether bond in PES molecular, the PES membrane is hydrophobilic. The hydrophobilic membrane can not apply for highly hydrophobic membrane separation process, such as membrane distillation (Khayet, 2003, pp.51-56), membrane adsorption and desorption (Lu, 2008, pp.180-190 and Yuan, 2008, pp.332-336), desalination (Ma, 2009, pp.5446-5450). In order to use PES membrane in highly hydrophobic membrane separation process, the hydrophobicity must be improved. Up to now, many significant techniques have been developed to fabricate the super-hydrophobic membrane, including plasma polymerization (Wang, 2006, pp.5493-5497), molding or template-based method (Feng, 2003, pp.800-802 and Luke, 2006, pp.3024-3028), electro-spinning (Kanga, 2008, pp.411-414), vapor deposition (Ma, 2005, pp.9742-9748 and Kanga, 2008, pp.411-414), phase separation (Ma, 2006, pp.39-42), etching (Narita, 2000, pp.594-597), Sol-gel method (Satoh, 2003, pp.327-332) and so on(Shi, 2005, pp.1005-1009 and Han, 2005, pp.20773-20778).

In this work, we report the super-hydrophobic PES membrane was gained through fluoroalkylsilane treatment by Sol-gel method. We investigated the different based membrane influence the hydrophobicity of super-hydrophobic PES membrane, and the structure of the super-hydrophobic membrane. Compared to the super-hydrophobic systems 
mentioned above, our preparation method is much simpler and easily scalable. SEM, AFM, XPS and ATR-IR indicated that the upmost layer exhibited both micro-structured surfaces and enriched fluorine.

\section{Experiment}

The solution was prepared at $80^{\circ} \mathrm{C}$ by dissolving PES into DMAc for $3 \mathrm{~h}$. The obtained solution was kept in vacuum for 30 min to degas. Then the PES solution was cast on a glass plate by a drawknife. The nascent membrane was remained for 30 seconds in air and then immersed in water coagulation bath for $24 \mathrm{~h}$ to precipitate the polymer. Last, the membrane was placed in the shade to dry for $8 \sim 10 \mathrm{~h}$.

Sol was prepared as described in literature (Guo, 2006, pp.761-766), $0.09 \mathrm{~mol} \mathrm{NH}_{4} \mathrm{OH}$ as a catalyst was dipped into $1.71 \mathrm{~mol}$ anhydrous ethanol, and kept stirring $30 \mathrm{~min}$ at $60^{\circ} \mathrm{C}$, and then dropped $0.026 \mathrm{~mol}$ TEOS. In order to ensure sol formation, the mix solution was stirring $90 \mathrm{~min}$ at $60^{\circ} \mathrm{C}$. Dodecafluoroheptyl-propyl-tirmethoxysilane (DPT-12) was dissolved in ethanol to form the fluorinated solution $(1 \mathrm{wt} \%)$ by the similar method of Sol. The process of preparing the super-hydrophobic PES membrane by Sol-gel from solution was described in Fig.1.

The contact angles of the super-hydrophobic membrane were measured using contact angle goniometer (JY-82, Chengde testing machine Co. Ltd., China) at ambient temperature with about $5 \mu \mathrm{L}$ water droplets. The characteristic element of the nanostructures formed on PES membrane was measured by X-ray photoelectron spectroscopy (XPS, Thermo K-Alpha). The chemical composition on membrane surface was analyzed by FTIR of TENSOR-37. The surface structure of the membrane was observed by SEM of FEI QUANTA Model-200. The morphology of the membrane and surface roughness were measured using atomic force microscopy (AFM, Agilent AFM/STM 5500). AFM was used to find the peak to trough height and the root mean square roughness $(\mathrm{rms})$ of the membranes to gauge the extent of their surface roughness. The rms is the standard deviation of height $(h)$ values with the specific area and is calculated as:

$$
\mathrm{rms}=\sqrt{\frac{\sum_{\mathrm{i}=1}^{\mathrm{n}}\left(\mathrm{h}_{\mathrm{i}}-\mathrm{H}\right)^{2}}{\mathrm{~N}}}
$$

Where $h$ is taken from the initial point ( $i$ ) to the final point $(n)$ measured on the membrane surface, $H$ and $N$ are the average of the $h$ values and the number of points within a given area, respectively.

\section{Results and discussion}

\subsection{Change of wettability on membrane surface}

The hydrophobilic ether bond was in PES molecular, so the PES membrane was weak hydrophobilic. As showed in Fig.2a, its water contact angle was about $75^{\circ}$. The hydrophobilic PES membrane was treated by sol-gel, the wettability changed from weak hydrophobilic to super-hydrophobic. As showed in Fig.2b, the water contact angle of the super-hydrophobic membrane surface reached $154^{\circ}$. Comparing to the based membrane, its contact angle was increased by $\sim 79^{\circ}$.

\subsection{Surface hydrophobicity in dependence of PES membrane-based}

The hydrophobic functional groups on the membrane surface were the fluorinated groups. The groups were grafted on membrane surface via dehydrating between- $\mathrm{OH}$ with $-\mathrm{Si}-\mathrm{OH}$. The $-\mathrm{OH}$ groups restricted the hydrophobicity of the membrane. The $-\mathrm{OH}$ groups on the membrane surface was the end group of PEG. If the PEG molecular weight was small, PEG would be emerged or dissolved in water of coagulation bath. That's to say, PEG molecular weight decided the amounts of the $-\mathrm{OH}$ group and the amounts of the fluorinated groups. As showed in Fig.3, the fluorinated. The contact angle increased with PEG molecular weight, and then decreased. That is that when molecular weight exceeded $10 \mathrm{k}$, the -OH groups was fewer than the same mass of low molecular weight of PEG.

The PES membrane-based was fabricated by phase inversion method. The upper layer (or air layer) and the glass layer have different pores structure for different separate phase speed. The otherness of hydrophobicity of two layers for different membrane was compared. As showed in Fig.4, though the two layers pores were different, the hydrophobicity of the fluorinated membrane was hardly same. The difference of the largest value and the smallest value about the upper and the glass layer was less than $10^{\circ}$.

\subsection{Chemical analysis of surfaces}

Fig.5 shows XPS spectra obtained from the PES membrane surfaces covered with the fluorinated layer. Four elemental compositions ( $\mathrm{Si}, \mathrm{C}, \mathrm{O}$ and $\mathrm{F}$ ) were clearly observed. The $\mathrm{F}$ at $690 \mathrm{eV}$ and $\mathrm{The} \mathrm{Si}$ at $104.08 \mathrm{eV}$ originated from the $\mathrm{Si}_{-} \mathrm{CF}_{3}$, which came from the DTP-12. The $\mathrm{Si}$ at $153.08 \mathrm{eV}$ and the $\mathrm{O}$ at $530.4 \mathrm{eV}$ components originated from the Si-O groups. The Si-O groups were caused by reaction between DTP-12 and the active groups on PES membrane treated by Sol, indicating that a fluorinated layer was formed on the PES microstructure surface.

As shown in Fig.6, the chemical compositions of the super-hydrophobic membrane's surface were analyzed by 
Fourier-Transform Infrared Spectrometers (FTIR). The peak in super-hydrophobic membrane's FTIR curve increased between $1000 \mathrm{~cm}^{-1}$ with $1400 \mathrm{~cm}^{-1}$, comparing to PES membrane-based FTIR curve. The peak at $1248 \mathrm{~cm}^{-1}, 1146 \mathrm{~cm}^{-1}$, $1104 \mathrm{~cm}^{-1}, 1020 \mathrm{~cm}^{-1}$ and $2957 \mathrm{~cm}^{-1}$ represented $-\mathrm{CF}_{3},-\mathrm{CF}_{2}$, $-\mathrm{CF}$, Si-O-Si and $-\mathrm{CH}_{3}$ groups, respectively, as reported in the literature. It is confirmed that the hydrophobic groups was low surface energy fluorinated groups.

\section{4 structure of the membrane's surface and cross-section}

As we know, the low surface energy material can increase the membrane hydrophobicity, but its contact angle was not exceeding $120^{\circ}$ yet. According to Wenzel and Cassie's theory, the surface hydrophobicity related with the micro-structure on surface. So the surface structure was analyzed by SEM and AFM. SEM image in Fig.7 illustrates the surface structure of the super-hydrophobic membrane. We can from Fig.7 that the super-hydrophobic membrane's surface had the crater-like structure. In order to realize clearly the exterior structure, 3D AFM topographical image of super-hydrophobic membrane clearly revealed that the membrane's surface consisted of protrusions of different height. The size of each protrusion was approximately the same size as each segment of the articulated features observed in Fig.8. The rms roughness of this membrane was $350 \mathrm{~nm}$.

As a membrane, the pores restricted the separability of the membrane. Being prepared the membrane-based, the pores were formed in phase separation process. In the fabrication process of super-hydrophobic membrane, the pores also changed with the treated condition. A key change was the pores' shrinkage. SEM images of top, underside and the whole cross-section of the super-hydrophobic membrane was shot as in Fig.9. The top image indicated that the membrane had two kinds of different pores. As showed in amplificatory Fig.9, the spongy pores were in membrane's cortex (Left amplificatory image), and the fingerlike pores were in intermediate layer (Left amplificatory image). In the water coagulation bath, the solvent and the non-solvent had different separate speed. The outside layer (or cortex) directly contacted water coagulation bath, and had rapidly separate speed. The outside layer formed the spongy pores. On the contrary, the inside (or intermediate and glass contacted side) had slowly separate speed for not contacting water as phase separation medium. The fingerlike pores were formed in inside layer.

\section{Conclusion}

In this work, the super-hydrophobic PES membrane was successfully prepared by Sol-gel method from DPT-12 solution. The super-hydrophobic surface of the PES membrane was clearly observed by SEM and AFM. The super-hydrophobic PES membrane has a higher surface roughness, which is caused by the formation of crater-like micro-texture. The rms roughness was found as higher as $350 \mathrm{~nm}$. The micro-structured surfaces and the low surface energy property jointly contributed the super-hydrophobicity of the membrane, which the water contact angle was about $154^{\circ}$. The Sol-gel method was a cheap and easily implemental method to prepare the super-hydrophobic membrane.

\section{Acknowledgment}

We are grateful for financial support from the Tianjin Nature Science Funding (No.08JCYBJC26300). This study is also supported by China Postdoctoral Science Foundation funded project (No.20080440686) and Open project of State Key Laboratory of Heavy Oil Processing, China University of Petroleum.

\section{References}

Barthlott, W., and Neinhuis C. (1997). Purity of the sacred lotus or escape from contamination in biological surfaces. Planta. 202, 1-8.

Feng, L., Li, S. H., Li, Y. S., Li, H. J., Zhang, L. J., Zhai, J., Song, Y. L., Liu, B.Q., Jiang, L., and. Zhu, D. B. (2002). Super-Hydrophobic Surfaces: From Natural to Artificial. Adv. Mater. 14, 1857-1860.

Feng, L., Song, Y. L., Zhai, J., Liu, B. Q., Xu, J., Jiang L, and Zhu, D. B. (2003). Creation of a Super-hydrophobic Surface from an Amphiphilic Polymer. Angew Chem. 42(7), 800-802.

Gu, Z. Z., Uetsuka, H., Takahashi, K., Nakajima, A., Onishi, H., Fujishima, A., and Sato. O. (2003). Structural Color and the Lotus Effect. Angew. Chem. 115, 922-925.

Guo, Z. G., Zhuo, F., and Liu, W. M. (2006). Preparation of bio-mimetic super-hydrophobic silica film by sol-gel technique. Acta. Chem. Sinica. 64(8), 761-766.

Han, J. T, Zheng, Y., Cho, J. H, Xu, X., and Cho, K. (2005). Stable super-hydrophobic organic-inorganic hybrid films by electrostatic self-assembly. J Phys Chem., B. 109, 20773-20778.

Kanga, M., Junga, R. and Kima, H. S. (2008). Preparation of super-hydrophobic polystyrene membranes by electro-spinning. J Colloids Surf. 2008, 313:411-414.

Khayet, M., and Matsuura, T. (2003). Application of surface modifying macromolecules for the preparation of membranes for membrane distillation. Desalination. 158,51-56.

Lu, J. G., Zheng, Y. F., and Cheng, M. D. (2008). Wetting mechanism in mass transfer process of hydrophobic 
Membrane gas absorption. Journal of Membrane Science. 308,180-190.

Luke, A., Connal, and Qiao, G. G. (2006). Preparation of Porous Poly (dimethylsiloxane)-Based Honeycomb Materials with Hierarchal Surface Features and Their Use as Soft-Lithography Templates. Adv. Mater. 18, 3024-3028.

Ma, M., Mao, Y., Gupta, M., Gleason, K. K., and Rutledge, G. C. (2005). Super-hydrophobic fabrics produced by electro-spinning and chemical vapor deposition. Macromolecules. 38, 9742-9748

Ma, Y., Ma, Y. M., Cao, X. Y., and Zou, H. (2006). Fabrication of super-hydrophobic plastic films via a simple method. Plastic. 35(5), 39-42.

Ma, Z. Y., Hong, Y., Ma, L. Y., and Su, M. (2009). Super-hydrophobic membranes with ordered arrays of nano-spiked micro-channels for water desalination. Langmuir. 25(10), 5446-5450.

Narita, M., Kasuga, T., and Kiyotani, A. (2000). Super water-repellent aluminum by electrolytic etching and chemical adsorption. Journal of Janpan Institute of Light Metals. 50(11), 594-597.

Satoh, K., and Nakazumi, H. (2003). Preparation of super-water-repellent fluorinated inorganic-organic coating films on nylon-66 by the sol-gel method using micro-phase separation. J Sol-Gel Sci. Tech. 27,327-332.

Shi, F., Wang, Z. Q., and Zhang, X. (2005). Combining a layer-by-layer assembling technique with electrochemical deposition of gold aggregates to mimic the legs of water striders. Adv Mater. 17, 1005-1009.

Wang, Q. J., Quan, Y. W., Zhang, J. S., and Chen, Q. M. (2006). Preparation of super water-repellent membrane by radiation-induced copolymerization. Surface and Coatings Technology. 200, 5493- 5497.

Yuan, J. K., Liu, X. G., Ozgeakbulut, Hu, J. Q., Suib, S., Kong, J., and Stellacci, F. (2008). Super-wetting nano-wire membranes for selective absorption. Nature nanotechnology (Letters). 3, 332-336.

Zhang, J. H., Sheng, X. L., and Jiang, L. (2009). The Dewetting Properties of Lotus Leaves. Langmuir. 25, $1371-1376$.

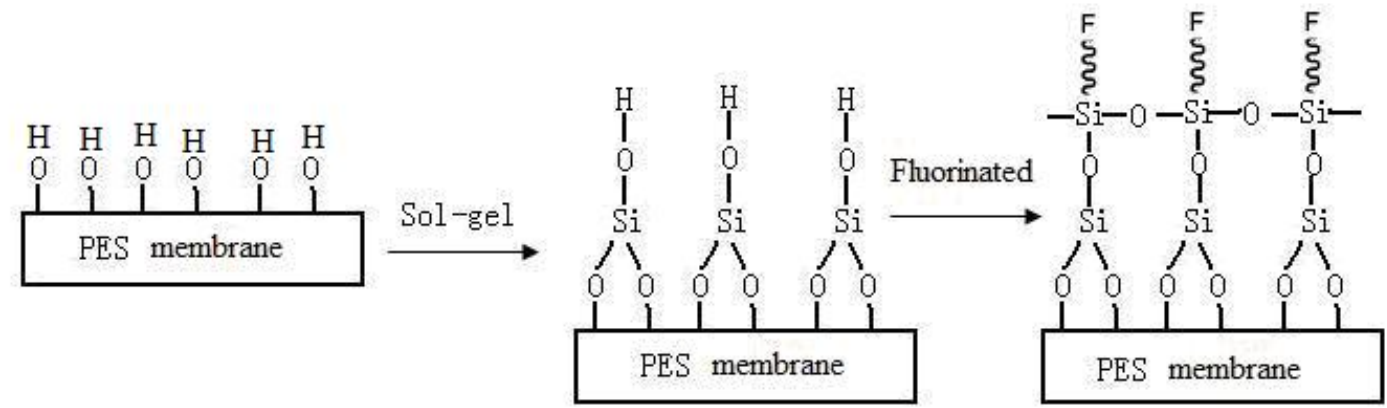

Figure 1. Scheme of fabrication of super-hydrophobic PES membrane by Sol-gel method

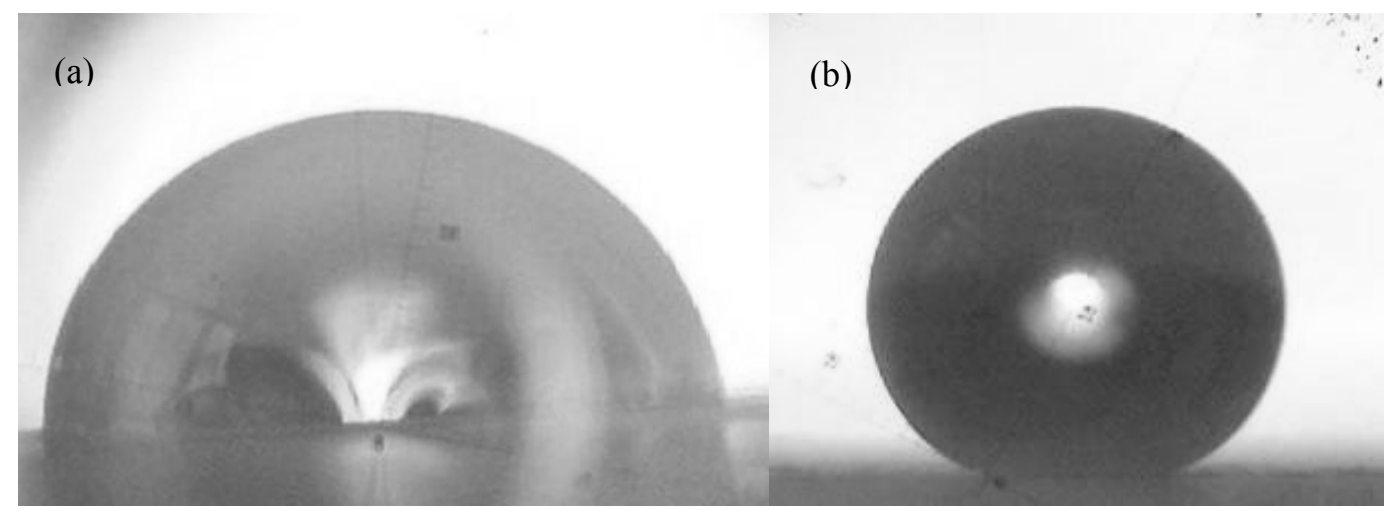

Figure 2. Water droplet $(\sim 5 \mu \mathrm{L})$ on corresponding membrane surface

a. PES membrane-based $\left(\sim 75^{\circ}\right)$; b. Super-hydrophobic PES membrane $\left(\sim 154^{\circ}\right)$. 


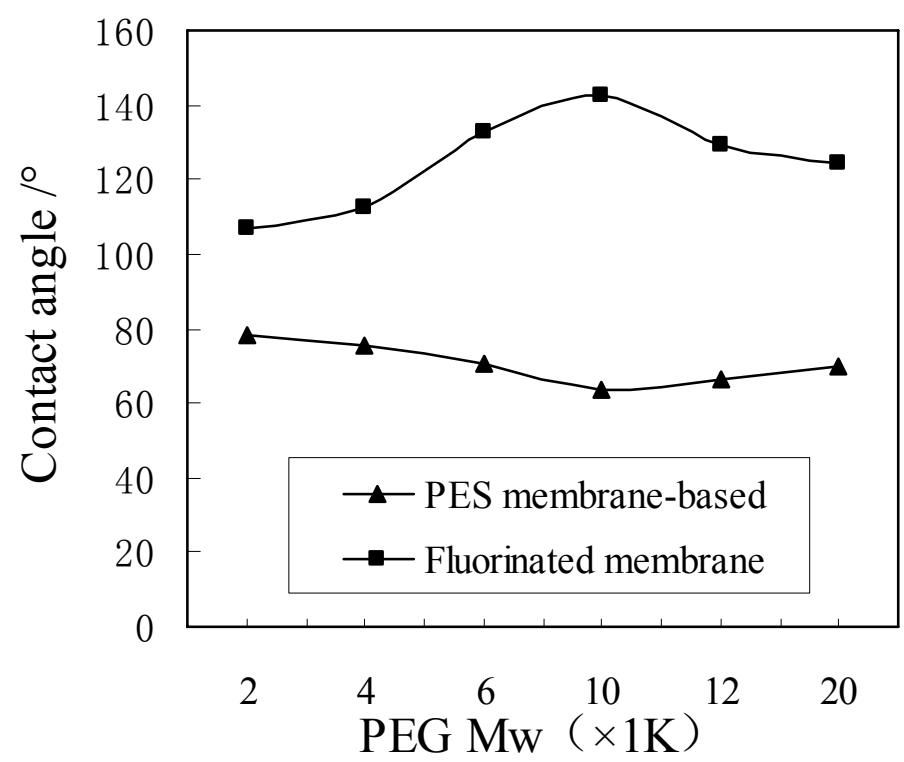

Figure 3. Hydrophobicity in dependence of PEG Mw in PES membrane-based

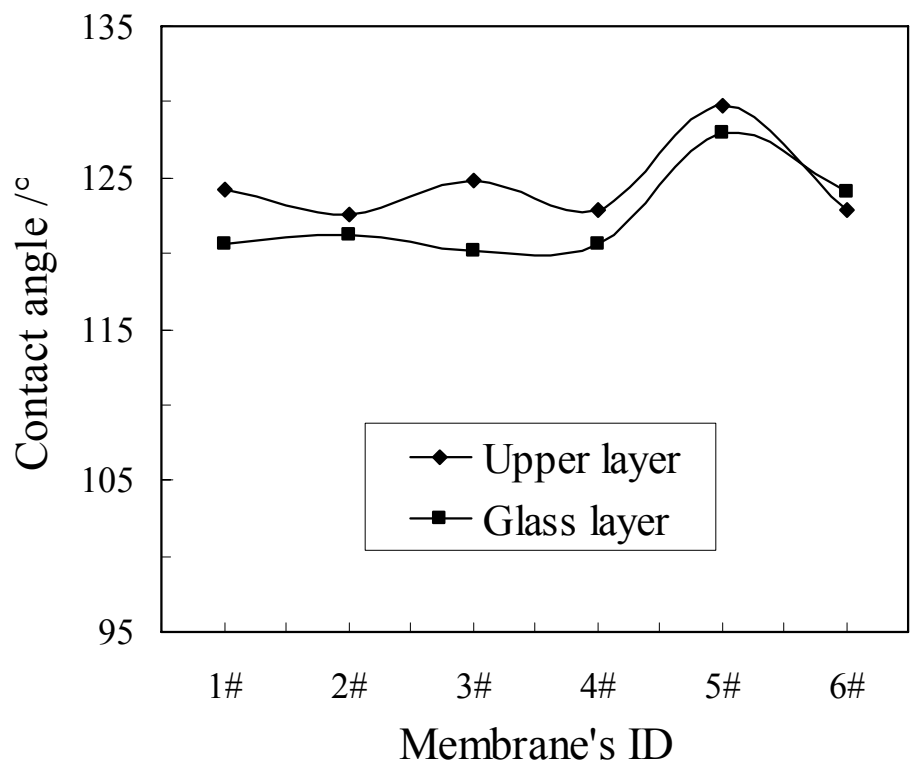

Figure 4. Otherness of hydrophobicity of two layers for different membrane 


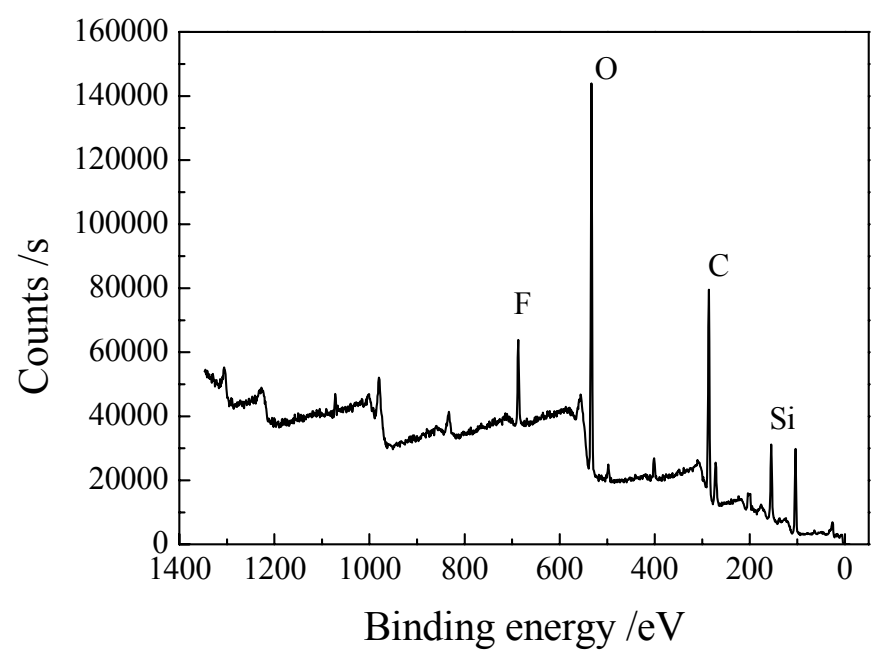

Figure 5. XPS spectra on super-hydrophobic membrane surface

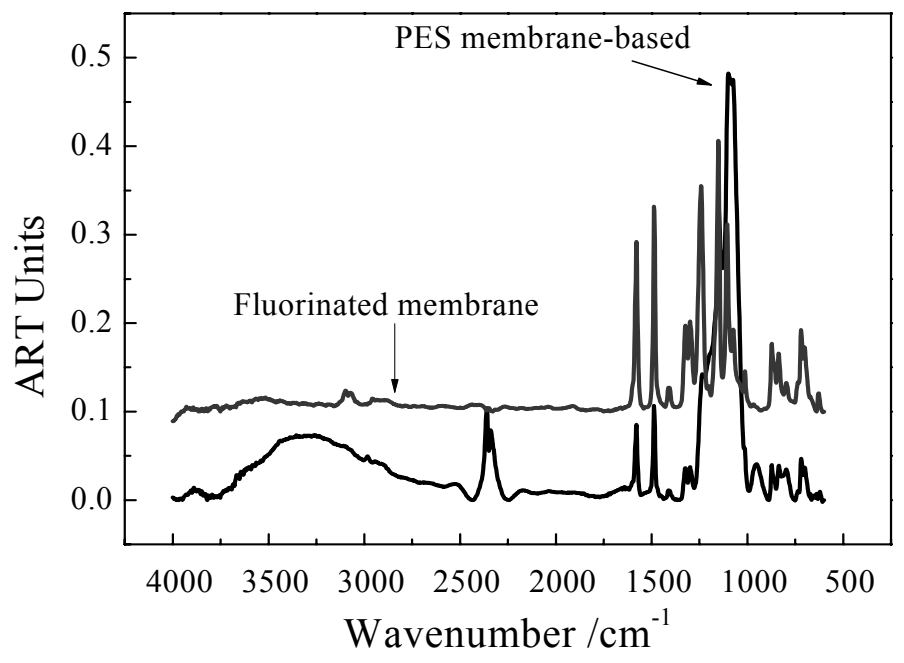

Figure 6. FTIR analysis of membrane surface

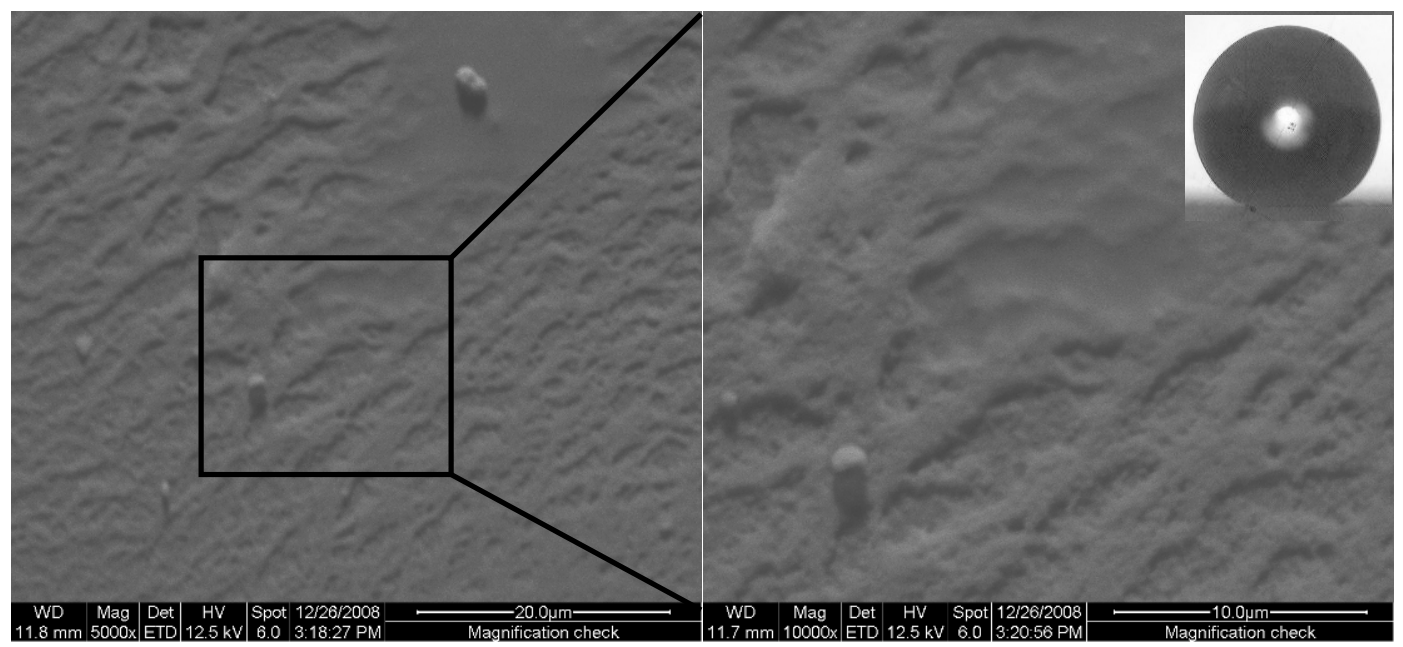

Figure 7. SEM image of PES super-hydrophobic membrane (Left) $\times 5000$, (Right) $\times 10000$ 


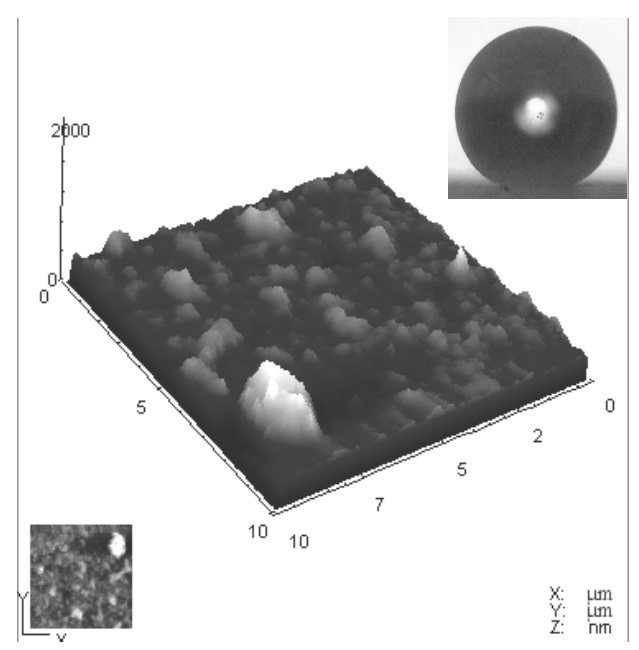

Figure 8. 3D AFM topographical image of super-hydrophobic membrane

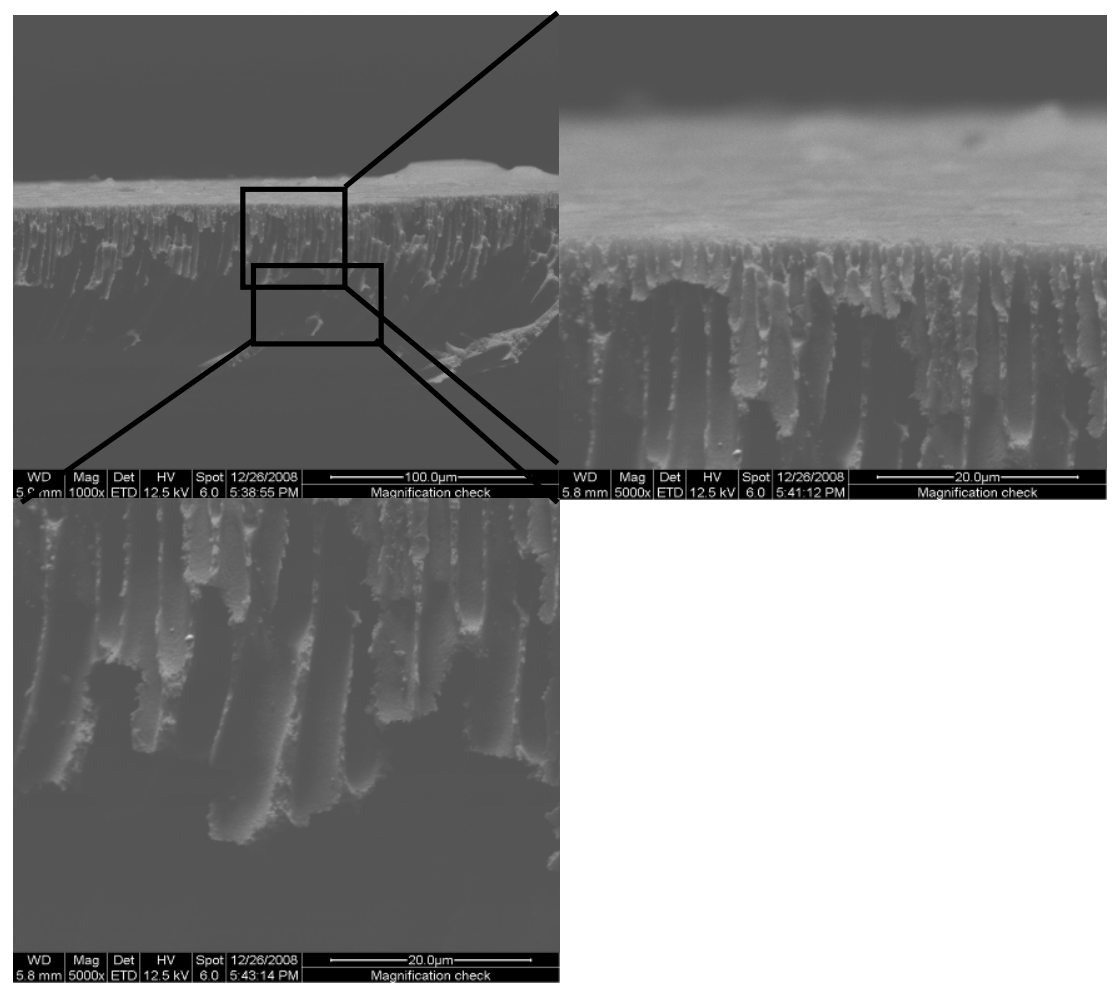

Figure 9. SEM image of the cross-section of the super-hydrophobic membrane 